\title{
PASSIVE CONTROL OF FLOW STRUCTURE INTERACTION BETWEEN A SPHERE AND FREE-SURFACE
}

\author{
Muammer OZGOREN ${ }^{1}$, Sercan DOGAN ${ }^{2}$, Abdulkerim OKBAZ ${ }^{3}$, Besir SAHIN ${ }^{4}$, Huseyin AKILLI ${ }^{5}$
}

\begin{abstract}
Flow characteristics for both a smooth and a vented sphere such as velocity vectors, patterns of streamlines, vorticity contours, stream-wise fluctuations, cross-stream velocity fluctuations and Reynolds stress correlations between a sphere and free-surface for various submerged ratio at $R e=5,000$ are studied by using dye visualization and the particle image velocimetry technique. Passive control of flow structure interaction between sphere and free surface was examined by using a modified geometry which has a $15 \%$ sphere diameter hole passing through the sphere equator. Both of the spheres were separately placed beneath the free surface with different positions from touching to the free surface to two sphere diameters below the free surface. It is demonstrated that reattachment point of the separated flow to the free surface varies for both of the sphere cases as the sphere position alters vertically through the water flow while the flow structure for the vented sphere occurs considerably symmetrical due to forming of a pair of counter-rotating ring vortices.
\end{abstract}

Keywords: Free-surface, Passive control, PIV, Sphere, Turbulence, Wake flow

\section{INTRODUCTION}

Fluid mechanics applications in between a free surface and sphere are encountered in the area of off-shore oil storage tanks, transporting of silicon and polymer, conveying of sediment materials in the river, spherical swimming bodies in the sea or rivers, sport balls, bombs, manned/unmanned submarine research vehicles, flow-structure interactions in aerodynamics and hydrodynamics. Result of fluid-structure interaction can cause important variations both on the bluff body and the flow structure. Therefore, there are numerous experimental, theoretical and numerical studies in the literature concerning with basic features of the flow structure around a sphere [1-20]. However, fewer studies were found about flow control using passive and active devices around a sphere given in the references and cited therein such as dimpled sphere [8-10], sphere with o-ring [10] and vented sphere [14-17]. Some of the other related studies on the flow control concerning with the present study can be found in the literature [20-28]. Suryanarayana and Prabhu [17] conducted experiments in water and wind tunnels on

\footnotetext{
${ }^{1}$ Assoc.Prof.Dr, Mechanical Engineering Department, email: mozgoren@selcuk.edu.tr

${ }^{2}$ Research Assistant, Mechanical Engineering Department, email: sercandogan@selcuk.edu.tr

${ }^{3}$ Project Assistant, Mechanical Engineering Department, email: abdulkerimokbaz87@gmail.com

${ }^{4}$ Prof.Dr, Mechanical Engineering Department, email: bsahin@cu.edu.tr

${ }^{5}$ Prof.Dr, Mechanical Engineering Department, email: hakilli@cu.edu.tr
} 
spheres in the Reynolds number range $6 \times 10^{3}$ to $6.5 \times 10^{5}$ to study the effect of natural ventilation on the boundary layer separation and near-wake vortex shedding characteristics. In the subcritical range of $\operatorname{Re}\left(<2 \times 10^{5}\right)$, ventilation caused a marginal downstream shift in the location of laminar boundary layer separation; there was only a small change in the vortex shedding frequency. In the supercritical range ( $\operatorname{Re}>4 \times 10^{5}$ ), ventilation caused a downstream shift in the mean locations of boundary layer separation and reattachment; their results showed significant axisymmetry in the presence of venting.

Most recently, passive control of vortical flow structure around a sphere by an o-ring for $\mathrm{Re}=5,000$ has been experimentally investigated. Visualization with Rhodamine dye and particle image velocimetry technique are performed to examine the flow characteristics such as instantaneous velocity fields, vorticity contours and time-averaged flow patterns of rms velocities in $\mathrm{x}$ and $\mathrm{y}$ directions, velocity fields and Reynolds stress correlations. $\mathrm{O}-$ rings with $2 \mathrm{~mm}$ and $3 \mathrm{~mm}$ diameters are located at the front side of the sphere having $42.5 \mathrm{~mm}$ diameter at angles of $45^{\circ}, 50^{\circ}, 55^{\circ}$ and $70^{\circ}$ to see suppression effect for dye experiment. It has been found from dye visualization and PIV results that the controlled flow structure results of the sphere with $2 \mathrm{~mm}$ o-ring at $55^{\circ}$ is the most effective [10]. The flow characteristics of a smooth sphere and a vent sphere located in a uniform flow for Reynolds number range $2500 \leq \operatorname{Re} \leq 10,000$ have been studied quantitatively using the particle image velocimetry technique and qualitatively with dye visualization. The flow phenomena in the downstream regions of the sphere increase the instability of the vortical flow structure significantly [13]. An experimental investigation of flow structures downstream of a circular cylinder and sphere immersed in a free-stream flow was performed for $\mathrm{Re}=5000$ and 10000 using qualitative and quantitative flow visualization techniques [14]. Flow data reveal that the size of wake flow region, the location of singular and double points, the peak values of turbulence quantities, such as Reynolds stress correlations, vorticity fluctuations and turbulent kinetic energy vary as a function of models' geometry and Reynolds Numbers. They stated that the concentration of small scale vortices is more dominant in the wake of the sphere than that of the cylinder.

The objective of the present work is to investigate the possibility of controlling flow structure in the downstream of the sphere by altering the position of the sphere from free-surface of water as well as the geometric modification in terms of a vent. In the literature, it has not been encountered any study that is related to the interaction between free-surface and sphere.

\section{EXPERIMENTAL SETUP}

Experiments were performed in a large-scale open water channel with a test section length of $8000 \mathrm{~mm}$ and a width of $1000 \mathrm{~mm}$ at the Department of Mechanical Engineering at Cukurova University, Turkey. To perform the present experimental study, the test section made from $15 \mathrm{~mm}$ thick transparent plexiglass sheet, which had a total height of $750 \mathrm{~mm}$, was filled with water to a level of only $450 \mathrm{~mm}$. Before reaching the test chamber, the water was pumped into a settling chamber and passed through a honeycomb section and a two-to-one channel contraction. An overview of experimental system of the sphere is shown in Figure 1. Free stream turbulence intensity of the flow is less than $0.5 \%$ in the range of the present Reynolds numbers, $\operatorname{Re}=\left(U_{\infty} D\right) / v$, based on the sphere diameter (D). Here, $v$ and $D$ are kinematics viscosity and diameter of the sphere (D), respectively. $U_{\infty}$ is the free-stream velocity taken as $117.6 \mathrm{~mm} / \mathrm{s}$ for 
$\mathrm{Re}=5,000$. The sphere with a diameter of $42.5 \mathrm{~mm}$ was made of plexiglass so that the laser light propagates easily from them. In addition, water cell segment of the sphere equator with a diameter of $38.5 \mathrm{~mm}$ and a wall thickness of $2.0 \mathrm{~mm}$ was created. It was filled with distilled water and had a total height of $8 \mathrm{~mm}$ in order to reduce largely the laser light deflection on the sphere. The sphere surface was highly polished to avoid effects of surface roughness. The laser sheet was located at $225 \mathrm{~mm}$ above the bottom surface of the channel while the water height $h_{w}$ was $450 \mathrm{~mm}$ in all cases. The Froude number based on the water depth $\mathrm{h}_{\mathrm{w}}$ was $F r=U_{\infty} / \sqrt{g h_{w}}=0.056$, which was subcritical flow region owing to the less than 1.0. To support the sphere in the water channel, a circular bar with a $5 \mathrm{~mm}$ diameter was connected to the sphere from the top. Disturbance effect of the support bar on the laser sheet location of the measurement plane that was observed by dye injection was negligible in the consideration of support diameter with respect to the sphere diameter. The solid blockage ratio of the sphere including support was $1.3 \%$. The vented sphere made of solid plexiglass and did not permit to pass the laser light. The ratio of the smooth sphere diameter $(D=42.5 \mathrm{~mm})$ to vented hole diameter $\left(d_{\text {hole }}=6.4 \mathrm{~mm}\right)$ is $d_{\text {hole }} / D=0.15$, which provides a vent area of $2.26 \%$. For dye visualization, dye ports with $0.7 \mathrm{~mm}$ diameter are located on the equator of the sphere at angle values with respect to the flow direction as $0^{\circ}, 70^{\circ}, 90^{\circ}, 110^{\circ}, 180^{\circ}$, $290^{\circ}, 270^{\circ}$ and $250^{\circ}$.

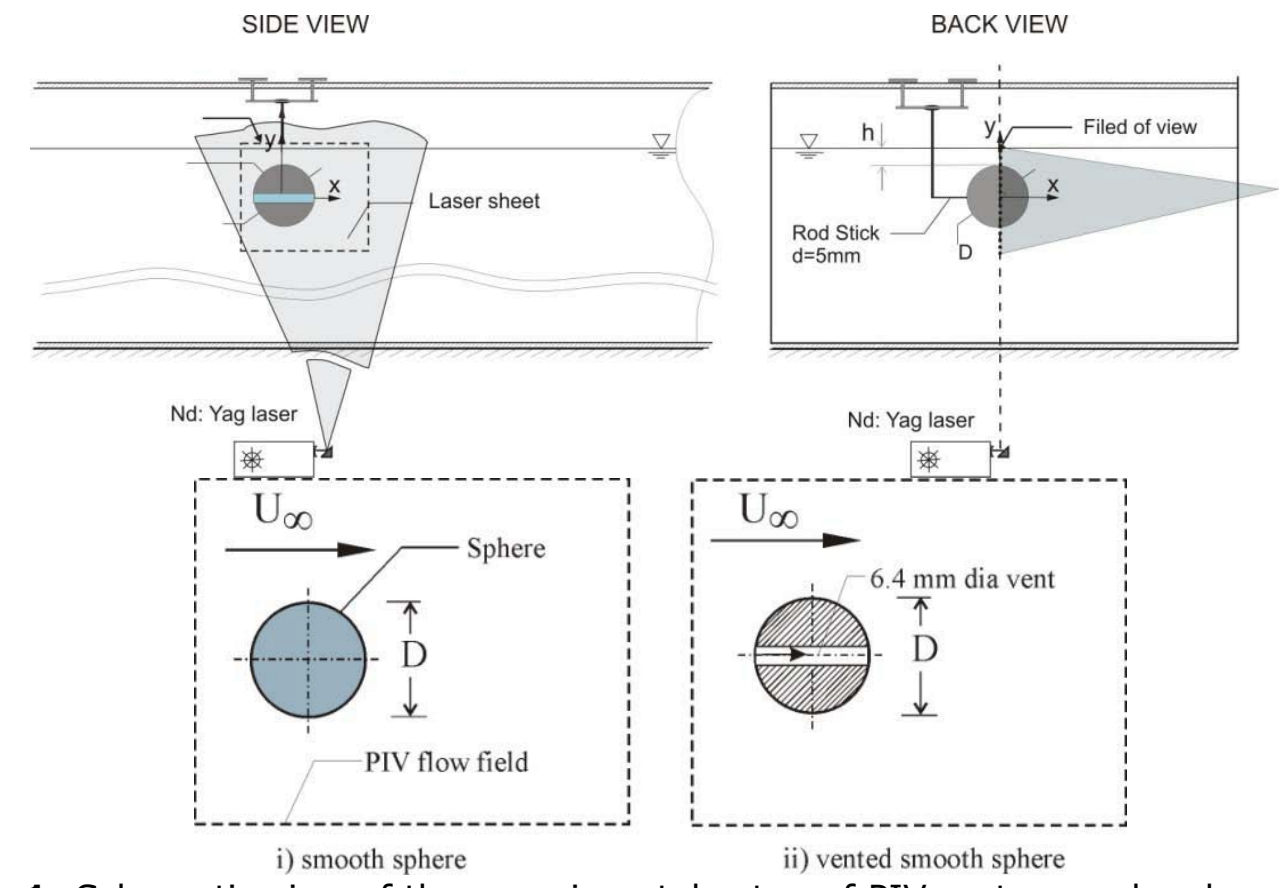

Figure 1: Schematic view of the experimental setup of PIV system and sphere position for a smooth and a vented sphere case

Nd:YAG laser was used to generate a laser sheet that was perpendicular to the axis for the sphere and the symmetry axis (i.e. equator of the sphere) was passed through them. A CCD camera having a resolution of $1,600 \times 1,186$ pixels was used to record the images. The seeding particles with a diameter of $10 \mu \mathrm{m}$ in the flow were silver metallic coated hollow plastic spheres. The densities of the particles and water are close enough so that the distribution of particles in suspension remains uniform for several hours. The high-image-density criterion was satisfied by ensuring that a minimum of approximately 20-30 particles was contained within the interrogation area. The illuminating laser sheet 
thickness in the flow field was approximately $1.5 \mathrm{~mm}$. As shown in Figure 1, the camera was mounted in a fixed position beneath the water tank. Dantec Flow Grabber digital PIV software employing the cross-correlation algorithm was used to compute the raw displacement vector field from the particle image data.

\section{RESULTS AND DISCUSSION}

Dye visualization representative images for the smooth and vented sphere case are presented in Figure 2. As it is seen, the separated and recirculating flow in the nearwake region with the help of visualizing are clearly seen for the sphere with laser illumination using the Rhodamine dye injection technique in the near wake region. Small scale vortices around the wake region designated by $A$ to $F$ are formed around larger vortices with a wavy appearance due to Kelvin Helmholtz instability. Formation of the spiral vortices begins to occur in the very close region of the sphere. As the flow travels in the downstream direction, the dimensions of the vortices increase around the bluff body. Then these vortices are shed from the periphery of the sphere directly to the inward wake region. The large eddies are formed at a regular frequency, and they produce pressure disturbances in the flow. The flow patterns in Figure 2 show that the laminar boundary layer separates at around $\theta=85^{\circ} \pm 5^{\circ}$, where $\theta$ is measured from the front stagnation point. The Reynolds number $R e=5000$, is well below critical, so the boundary layer on the sphere is laminar. Shedding shear layer becomes unstable due to the Kelvin-Helmholtz instability caused by the large velocity difference at the interface between the free-stream and sphere wake flow regions. Thereafter, the laminar shear layer turns into a powerful turbulent flow structure. Several vortex-ring shaped protrusions appear as an indication of the shear-layer instability along the borders between the wake and free stream regions, as also observed by Jang and Lee [3]. For the cases of $\mathrm{h} / \mathrm{d}=0$ and 0.1 in Figure 2, shedding vortices from the bottom shoulder of the sphere which have high rate interactions travel in the direction of the main flow to merge with the free-surface. As it is clearly seen from the top three images in Figure 2, the wake region of the sphere with the vent is shortened in size compared with the results of the smooth sphere due to the occurring of jet flow through the vent. The jet flow exiting from the vent moves in longitudinal direction and stirs the wake flow region to increase the entrainment to a higher level as clearly seen for the right column images. As seen from dye visualization in Figure 2 and instantaneous flow patterns in Figures 3 and 4, the wake becomes increasingly unstable for the submerged depths between $0 \leq h / D \leq 0.50$ and the vortex shedding keeps its axisymmetry. The vortices become highly threedimensional with quasi periodic shedding apparent in images taken at the equator plane of the sphere with and without vent.However, this patterns could be changed considerably for a vented sphere case.

Figures 3 and 4 show comparison of instantaneous velocity fields $V$ and $\omega^{*}$ around the smooth sphere (left column) and vented sphere (right column) for $\operatorname{Re}=5,000$. Instantaneous vorticity contours of the wake structure is normalized as $\omega^{*}$ (i.e., $\left.\omega^{*}=\omega \mathrm{D} / \mathrm{U}_{\infty}\right)$. All figure dimensions are normalized with the sphere diameter designated as $x / D$ and $y / D$. Layers of positive and negative vortices are displayed as solid and dashed lines, respectively. The minimum and incremental values of the flow patterns are shown on each image in Figures A concentrated vortex cluster from the bottom shoulder of the spheres for $h / D=0$ and 0.1 expands along the shear layer in the downstream direction. As seen from submerged depth until $h / D=0.5$, the vortices on the bottom shoulder of the 


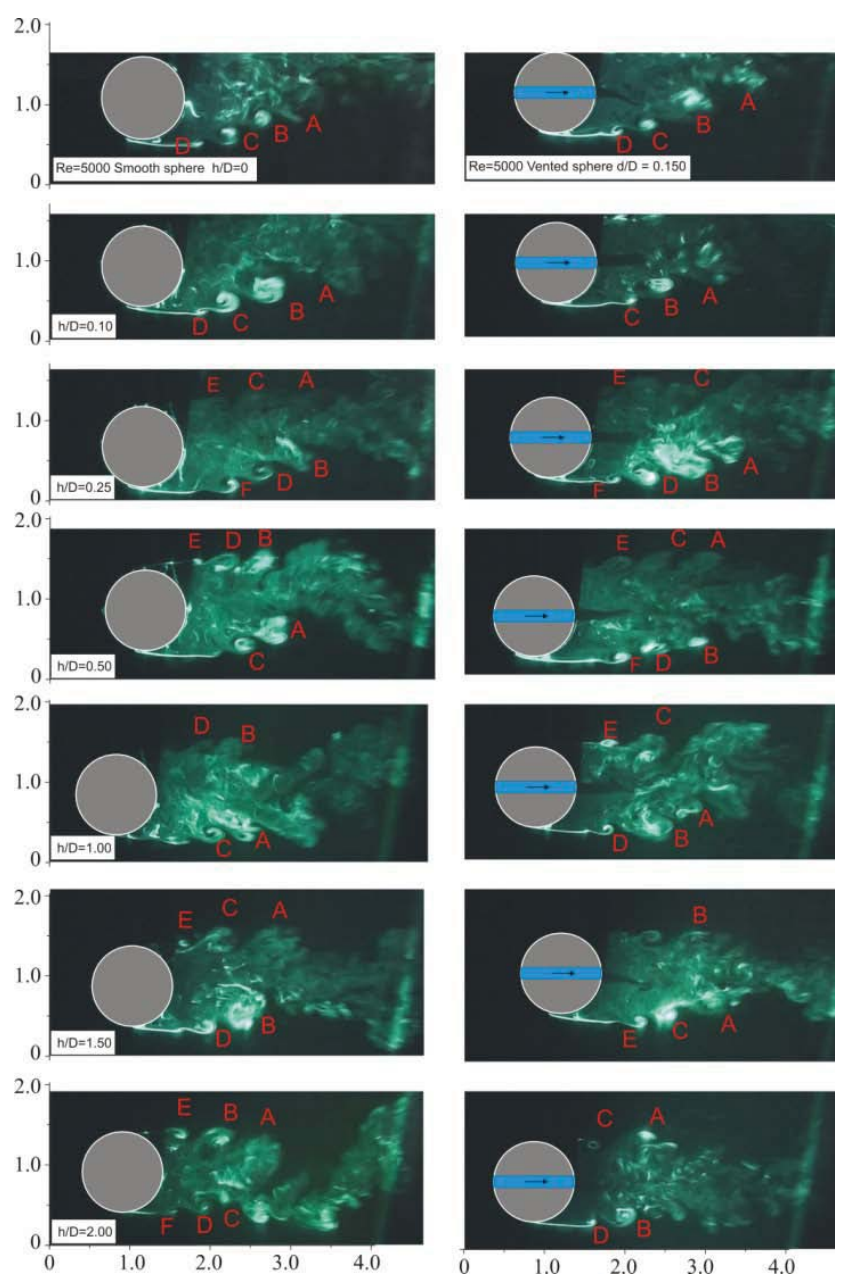

Figure 2: Comparison of flow visualization of flow structure with laser illumination of Rhodamine dye injection technique around the smooth sphere (left column) and vented sphere (right column) at $\operatorname{Re}=5,000$.

sphere can shed at considerably different times causing small asymmetries in the wake pattern. The flow regime is unstable and restricted in downstream of the sphere around three sphere diameters region. The length of the wake region increases with increasing submerged depth of the sphere for both of the sphere cases. In addition, at higher h/D $>0.5$, the distortion effect of the vent is relatively smaller and suppression of the vortical structure is medium level, thus leading to relatively higher concentrations. Even though small-scale Kelvin Helmholtz's vortices are clearly shown for dye visualization results, PIV results display more rounded vortices. However, numerous eddy vortices occur due to the three dimensional and complex flow structures. Jet flow through the ventilation hole make the flow structure more symmetric and increase the fluctuations in the base region of the sphere for $h / D>1.0$. The vortices produced from the flow separation around the periphery of the sphere have a tendency to move inwards because of the lower pressures prevailing within the wake. This situation is counter-balanced by the growing wake size, which shifted the vortex centerline outwards. Regarding the onset and development of small-scale vortical structures in the separating shear layer, regions of low-level vorticity concentrations are discernible in the patterns of instantaneous vorticity for all patterns. Large-scale of swirling patterns of velocity vectors are evident in the downstream of the 
sphere and corresponding patterns of vorticity shows that the shear layer is pushed to the base region causing a well defined concentration of vorticity as seen in all images in Figures 3 and 4 . The wake region accommodates velocity vectors with very small magnitude in the downstream region of the smooth and vented sphere, which are the source of small-scale secondary vortices, as seen in Figures 3 and 4 . The streamwise separation of successive vorticity peaks in the near wake region for the smooth sphere is larger than that for the vented one. The flow is three-dimensional, and shedding vortices convey fresh fluid into the wake flow region, magnifying the entrainment processes, which occurs with higher order of magnitude for the vented sphere. However, it is clear that there is only a small change on the flow patterns in the presence of ventilation for $h / D>1.0$. On the other hand, Suryanarayana and Prabhu [17] stated that the observed drag reduction of wake unsteadiness, presumably caused by the stabilization/weakening of the randomly rotating 3-D vortical structure of the smooth sphere, which was also observed in the present study. As seen in Figure 4, secondary vortices forms in the wake region due to jet flow through the vent. It may be noted that any reduction in the wake unsteadiness may be expected to result in drag reduction. The modified flow structure of the near wake of the vented sphere may be characterized by a pair of counter-rotating ring vortices, which have the effect of aerodynamically streamlining the sphere as expressed by Suryanarayana and Prabhu [17]. As pointed by Suryanarayana and Prabhu [17], the effect of natural ventilation can be categorized in two broad regimes as weak and strong interaction regimes. At subcritical Reynolds number $\left(\operatorname{Re}<4 \times 10^{5}\right)$, the weak interaction regime occurs and the broad features of the basic unvented sphere are largely unaltered despite the larger addition of mass in the near wake. Strong interaction is promoted by the closer proximity of the inner and outer shear layers at the supercritical $\mathrm{Re}$, which results in a modified/weakened and steady near-wake flow characterized by reduced unsteadiness. Sheridan et. al. (1997) studied on flow past a cylinder beneath a free surface which gives rise to fundamental classes of near wake structure creating distinctly different from the wake of a completely submerged cylinder. They demonstrated that the instantaneous vorticity flux on either side of this jet is rapidly balanced immediately after the onset of separation from the free surface. The jet of fluid passing over or under the sphere exhibited a number of possible states including: attachment to the free surface; attachment to the sphere surface; and an intermediate state in between as in the experimental results of Sheridan et al.[26].

For the cases of $h / D=0.1$ and 0.25 , instantaneous patterns of vortices and velocity vectors indicate that wake flow regions shrink in size in the stream-wise direction while the main flow travels further downstream. The length of reattachment of the wake region with the free surface is shorter for the sphere with the vent. The location of reattachment point is not clearly defined by the instantaneous velocity vectors $V$, because the point of the reattachment moves forward and backward randomly. Through the observation of an animation of instantaneous images, it is seen for the sphere with the vent case that small-scale concentrations of vorticity convey the fresh fluid into the wake flow region magnifying the entrainment between the free-stream and wake flow regions. Instantaneous locations of reattachment points move backward and forward in the direction of the free stream flow due to the instability of the vortical flow structure. Flow patterns of vorticity show distinctive small-scale concentrations embedded in the largerscale structures. They induce new types of elongated large-scale structures in the nearwake region, thus extending recirculation bubble as seen at the top images of Figure 4 . 
In fact, these identifiable structures of vorticity exist even at region well downstream of the free-stagnation point as seen for the cases $h / D>1.0$.

Comparison of the normalized flow patterns of the smooth sphere (left column) and the sphere with a vent (right column) for the time-averaged velocity field $\langle\mathrm{V}\rangle$, streamline patterns $\langle\psi\rangle$, time-averaged vorticity $\left\langle\omega^{*}\right\rangle$ (i.e., $\left\langle\omega^{*}\right\rangle=\langle\omega \mathrm{D} / \mathrm{U}\rangle$ ) rmc (rnnt mean square) velocity fluctiratinn mntours of the stream-wise velocity $\left\langle u_{r m s} / U_{\infty}\right\rangle$ and cross-stream velocity $\left\langle v_{r m s} / U_{\infty}\right\rangle$ are displayed in Figures 5-9. For these and other figs, the minimum and normalized incremental values of the patterns are given on each image.

Variations of the time-averaged velocity fields $\langle\mathrm{V}\rangle$ and vorticity contours $\left\langle\omega^{*}\right\rangle$ depending on the sphere locations beneath the water free-surface and effect of the ventilation for $\operatorname{Re}=5,000$ are displayed in Figures 5 and 6 . The time-averaged velocity fields $\langle\mathrm{V}\rangle$ indicate that vortex formation occurs immediately adjacent to the base of the sphere and those shedding vortices immigrate in the direction of the free stream flow to merge by the free-surface of the water channel. It is obviously seen that near wake formation changes periodically due to the jet flow from the vent into the wake flow region. The jet flow from the vent goes along the ventilation axis and slightly changes the direction toward the free-surface by dividing the wake region for $\leq 0 / D \leq 0.25$.

Having the flow jet through the vent for the right column images in Figure 6, wake flow region of the sphere is activated. It is apparent from the instantaneous and timeaveraged images that the jet flow through the vent can substantially affect the onset and development of large-scale vortical structures in the near wake region, relative to those occurring from the smooth sphere for $h / D<0.5$. A pair of vortices for the case of the sphere with the vent is developed in the wake region due to the jet flow emanating from the vent. For $h / D=2.0$, two symmetric vortices rotating in opposite direction are clearly seen in both cases in Figure 6 . Time-averaged positive and negative vorticity layers are approximately equal and symmetric with each other. For the sphere with the vent, flow structures are restless due to the continuous developing flow process due to the vent. Entrainment process due to the high rate of circulatory flow motion on vortical flow structure is extremely high engender energetic wake flow structure. As it is seen from time-averaged flow data for the sphere with the vent, distributions of flow patterns in the wake-flow region is shortened in size in the longitudinal direction and slightly narrowed in size lateral direction comparing with the smooth sphere.

As seen in Figure 6, time-averaged vorticity images $\langle\omega *>$ of the sphere with and without a vent reveal that the detailed instantaneous structure of the small-scale vortices and far downstream part of the Kármán vorticity street disappear completely due to the unsteady flow structure and alternating direction of the shedding vorticity from both sides of the spheres for the sphere with and without a vent cases. In the base region, two separated shear layers with oppositely signed vortices interact with each other more Flow patterns of the time-averaged stream-wise velocity fluctuations $\left\langle u_{r m s} / U_{\infty}\right\rangle$ and cross-stream velocity fluctuations $\left\langle\mathrm{v}_{\mathrm{rms}} / \mathrm{U}_{\infty}\right\rangle$ depending on the sphere locations beneath free-surface of the water and effect of the ventilation for $\operatorname{Re}=5,000$ are shown in Figures 7 and 8.

Flow patterns of the time-averaged stream-wise velocity fluctuations $\left\langle\mathrm{u}_{\mathrm{rms}} / \mathrm{U}_{\infty}\right\rangle$ and cross-stream velocity fluctuations $\left\langle v_{r m s} / U_{\infty}\right\rangle$ depending on the sphere locations beneath free-surface of the water and effect of the ventilation for $\operatorname{Re}=5,000$ are shown in Figures 7 and 8. 

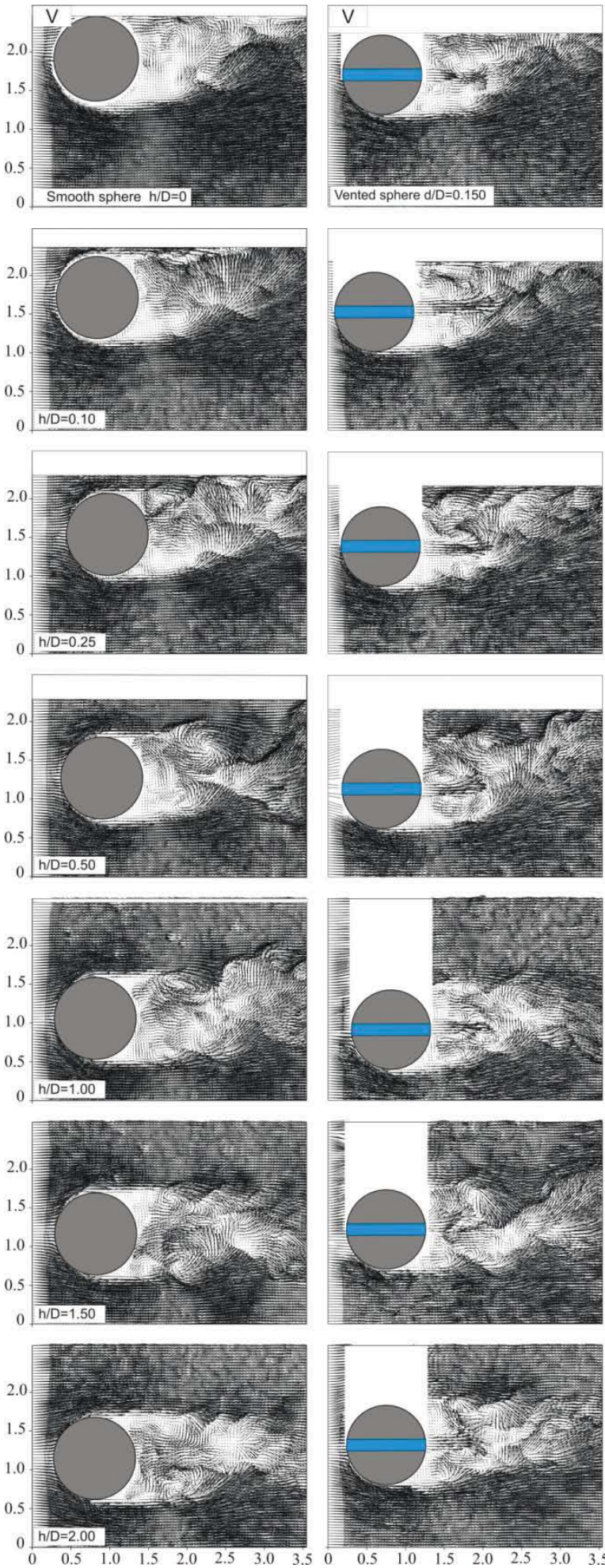
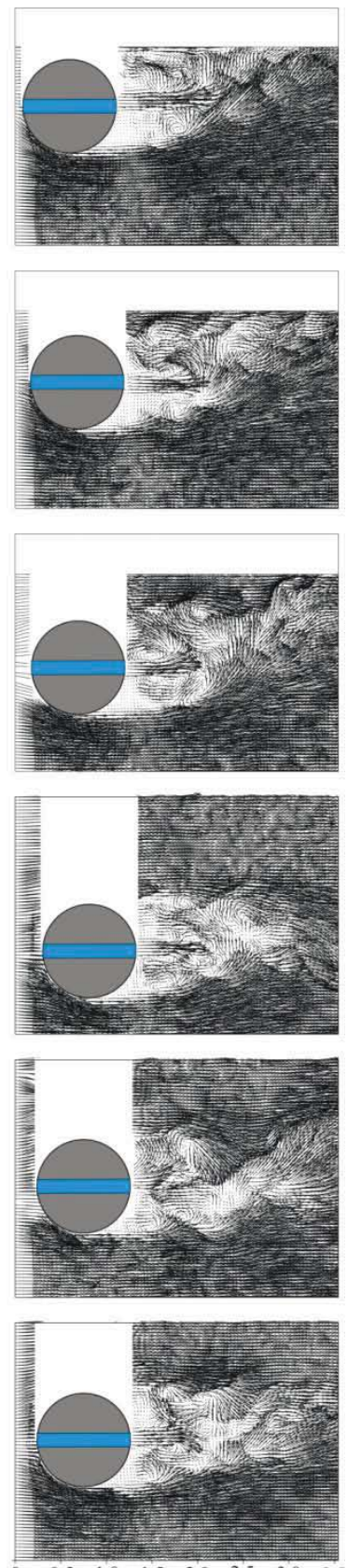
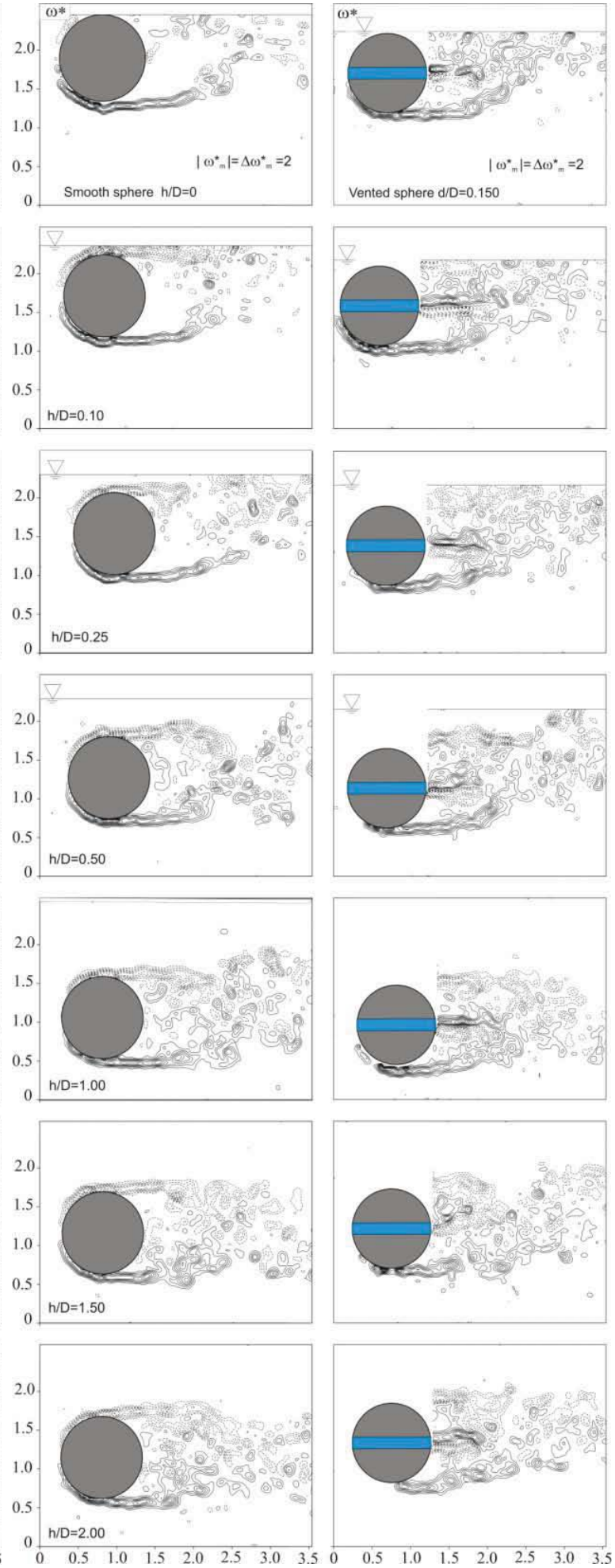

Figure 3: Variation of the instantaneous Figure 4: Variation of the instantaneous velocity fields $\mathrm{V}$ depending on the sphere vorticity contours $\omega^{*}$ depending on the locations beneath the water free-surface sphere locations beneath the water freeand effect of the ventilation for $\operatorname{Re}=5,000$. surface and effect of the ventilation for $\operatorname{Re}=5,000$. 

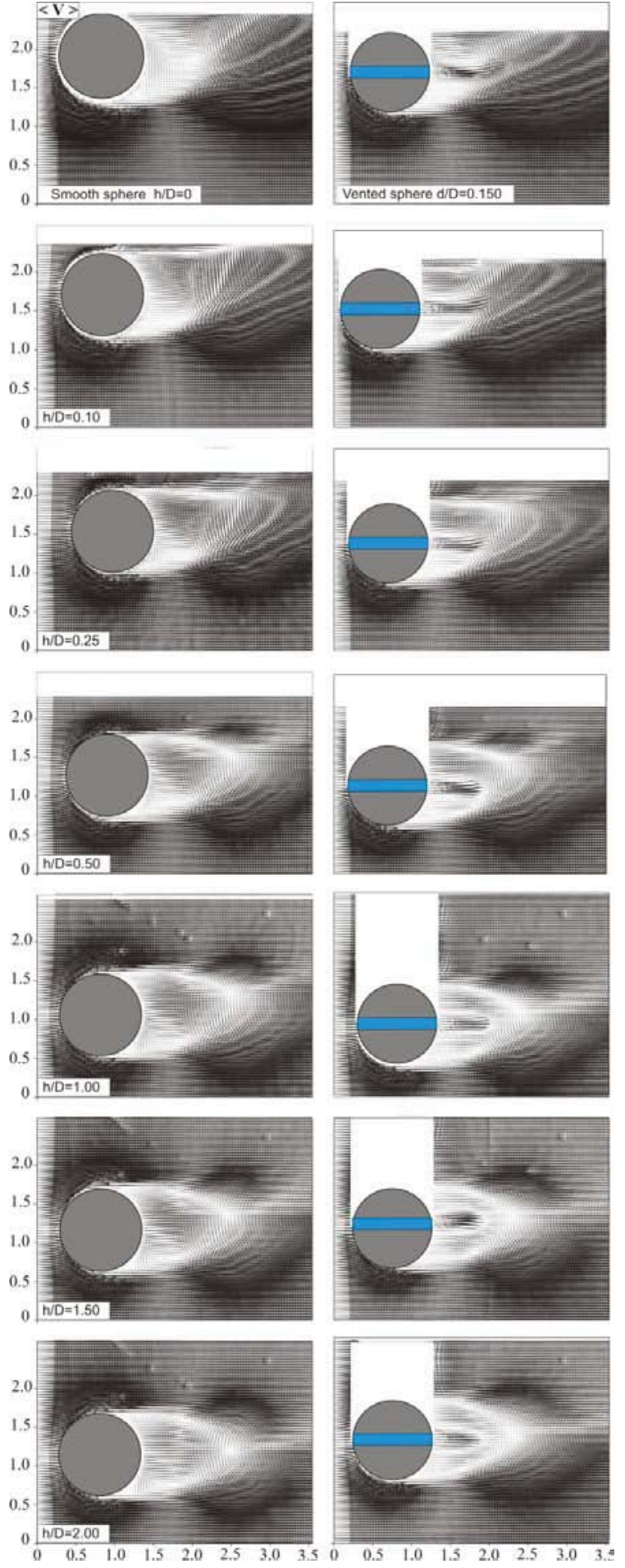

Figure 5: Variation of the time-averaged velocity field $\langle V\rangle$ depending on the sphere locations beneath the water freesurface and effect of the ventilation for $\operatorname{Re}=5,000$.
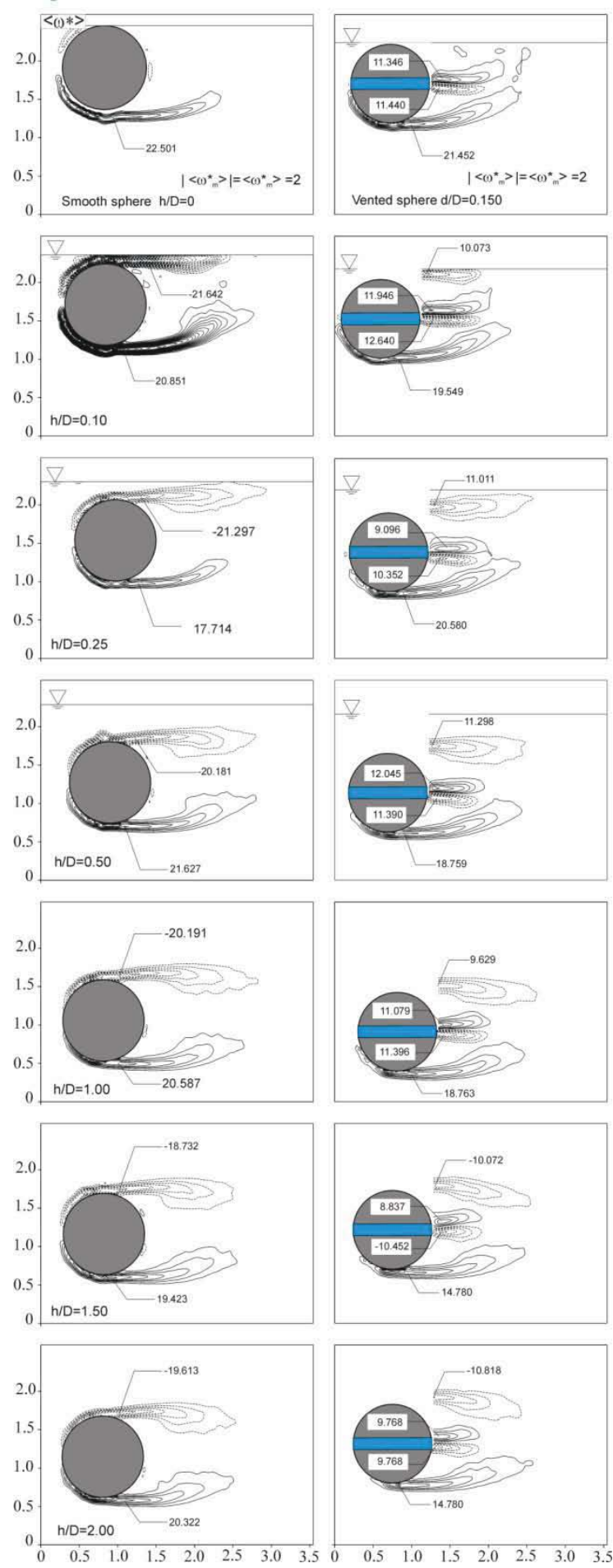

Figure 6: Variation of the time-averaged vorticity contours $\left\langle\omega^{*}\right\rangle$ depending on the sphere locations beneath the water free-surface and effect of the ventilation for $\operatorname{Re}=5,000$. 

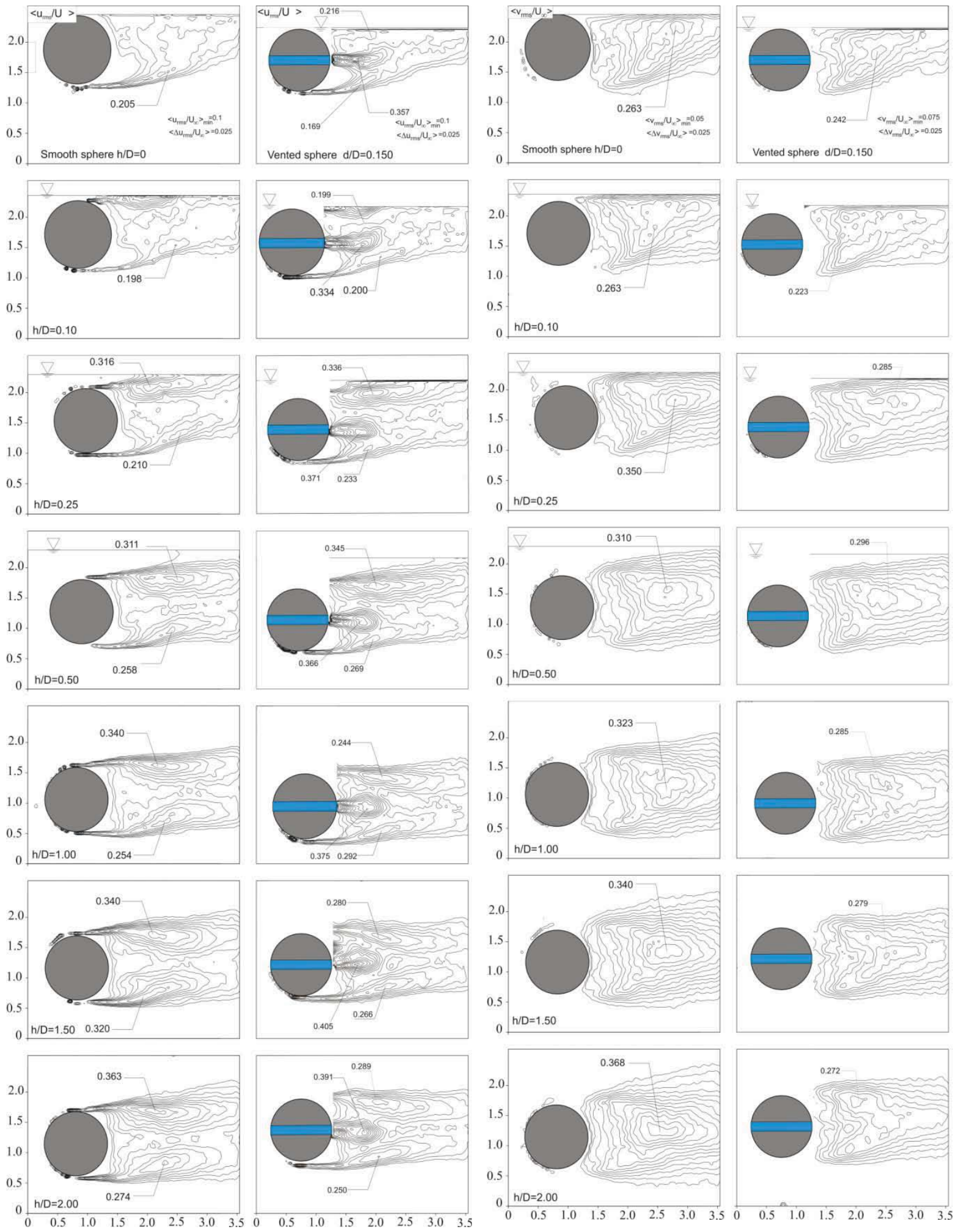

Figure 7: Variation of the time-averaged Figure 8: Variation of the time-averaged stream-wise velocity fluctuations $\left\langle\mathrm{u}_{\mathrm{rms}} / \mathrm{U}_{\infty}\right\rangle$ depending on the sphere $\left\langle\mathrm{v}_{\mathrm{rms}} / \mathrm{U}_{\infty}\right\rangle$ depending on the sphere locations beneath the water free-surface locations beneath the water free-surface and effect of the ventilation for $\operatorname{Re}=5,000$. and effect of the ventilation for $\operatorname{Re}=5,000$. 
The rms stream-wise velocity fluctuations patterns $\left\langle u_{r m s} / U_{\infty}\right\rangle$ have detectable double peaks at almost equal distances in the upper and lower wake regions of the centerline for the smooth sphere whereas they have three peaks, one of them emanating from the vent, for the case of the sphere with the vent for $h / D>1.0$. For the contours of $\left\langle\mathrm{v}_{\mathrm{rms}} / \mathrm{U}_{\infty}\right\rangle$, the cross-stream fluctuations are associated with the process of formation of the large-scale vortical structures. Peak values of the cross-stream fluctuations $\left\langle\mathrm{V}_{\mathrm{rms}} / \mathrm{U}_{\infty}\right\rangle$ for the sphere with the vent depending on the submerged depth are less than the smooth one as shown in Figure 8. In addition, the distance between the sphere base and the maximum point location for all submerged ratios $h / D$ decreases for the sphere with the vent. For example, the maximum values of the $\left\langle v_{\mathrm{rms}} / \mathrm{U}_{\infty}\right\rangle$ contours for $\mathrm{Re}=5,000$ on the centerline of the wake take place nearly at approximately $1.26 \mathrm{D}$ and $0.81 \mathrm{D}$ for the sphere without and with the vent at $h / D=2.0$, respectively.

The cross comparison of the results reveals double peaks for $\left\langle\mathrm{u}_{\mathrm{rms}} / \mathrm{U}_{\infty}\right\rangle$, while a single peak is seen in $\left\langle v_{r m s} / U_{\infty}\right\rangle$ with the maximum occurrence around the axis for both of the sphere cases.

Comparison of time-averaged streamline topology $\langle\Psi\rangle$ around the smooth sphere (left column) and vented sphere (right column) for $\mathrm{Re}=5,000$ is displayed in Figure 9 . The junction point of the streamlines separated from the surface of the sphere is denoted with " $\mathrm{S}$ ". This junction point is called as Saddle point $\mathrm{S}$ which shows the merging point of the shear layers emanating from both sides of sphere. Locations of foci designated with "F1 and F2" that are rolling in the clockwise and in counter clockwise direction on with respect to the sphere central axis exhibit well-defined critical points. It can be concluded that the flow around spheres is three dimensional. The separated flow regions are more compact for the sphere with the vent since the jet flow goes through the vent directly into the wake region. Because of this jet flow, the size of foci are very small in the near wake of the sphere. The foci, F1 and F2 are almost symmetrical as the submerged ratio of the sphere increases. Two saddle points occur for the jet flow as S1 and main flow as $\mathrm{S} 2$ as shown at the right column images of Figure 9, specially for $\mathrm{h} / \mathrm{D} \geq 0.25$. The timeaveraged streamline pattern shapes extended upstream to near base region of the body are elliptical and have a limited spiral cycle. Comparison of the time-averaged patterns shows that flow structures of the wake are almost equally symmetrical with respect to the centerline of the smooth and vented sphere. For the sphere with the vent, saddle point S1 becomes closer to the base of the sphere due to the increased momentum transfer through the ventilation. For smooth sphere case, two foci $F 1$ and F2 occur while flow structure of the vented sphere case exerts four foci as F1, F2, F3 and F4 due to the jet flow through the ventilation hole. Two saddle points S1 and S2 for the vented sphere case occur owing to the presence of jet flow. Viewing each of the flow fields of Figure 6 for $h / D=0$; it is evident that well-defined reattachment of location is a common feature which is indicated by the vertical arrow immediately adjacent to the free-surface. As seen from the time-averaged streamline patterns in Fig 6., the reattachment distances from the base of the smooth sphere were respectively determined as $1.90 \mathrm{D}$ and $1.16 \mathrm{D}$ for directly and vigorously, resulting in a substantial cancellation of the time-averaged vorticity. Two large-scale recirculation vortices formed downstream of the sphere are nearly symmetrical with respect to the wake centerline, even though instantaneous vortex structures are unsteady and irregular. These well defined circulating large-scale vortices dominate the flow structure in the near-wake downstream of the sphere, and these results are in a good agreement with those of other studies (Hadzic et al.[2], Jang 
and Lee[3], Leder and Geropp[4]). As it is seen from cross-comparison of the timeaveraged velocity fields $\langle\mathrm{V}\rangle$ and vorticity contours $\left\langle\omega^{*}\right\rangle$, distributions of the flow patterns clearly indicate that the wake flow area for the sphere without vent is smaller than that for the sphere with the vent. $h / D=0$ and 0.1 . Those for the sphere with the vent were found to be around $1.54 \mathrm{D}$ and $1.33 \mathrm{D}$ for $\mathrm{h} / \mathrm{D}=0$ and 0.1 , respectively. For $h / D=0$ configuration, a primary circulating bubble or developing focus which are designated as $F 1$ is formed and covers whole the wake flow region. For the case of h/D $=0.25$, the wake region with foci $\mathrm{F} 1$ and $\mathrm{F} 2$ are identifiable and then the saddle point $\mathrm{S}$ is formed just beneath the free-surface for the smooth sphere. Furthermore, each of these developing and primary cells has a stable focus that is the streamlines spiral towards inward the central points of foci. The length of the wake flow region in the stream-wise direction is varied as a function of $h / D$. The effective length of the jet flow decreases with increasing the submerged depth of the sphere. The saddle point S1 on the time-averaged streamline topology generally forms earlier for the vented sphere case. For example at $h / D=2.0$, the non-dimensional wake lengths (L/D) measured from the base of the sphere to the saddle point $S 1$ in the averaged streamline topology $\langle\Psi\rangle$ are approximately $1.21 \mathrm{D}$ and $1.07 \mathrm{D}$ for the smooth sphere and vented sphere, respectively. Here, $L$ is the distance between the sphere base and saddle point $S 1$. The $L / D$ values for the sphere without the vent were respectively determined as $0.98,1.5,1.18$ and 1.19 for the submerged depth ratios of $h / D=0.25,0.50,1.0$ and 1.50 while those for the sphere with the vent were, in sequence, $0.72,0.98,1.08$ and 1.06 . Finally, it can be interpreted that the drag force could decrease as the saddle point becomes closer to the sphere.

\section{CONCLUSIONS}

The sphere is placed from the free surface touching position to two sphere diameters below the free surface with different locations. Dye visualization, velocity vector fields and corresponding vorticity contours, streamline topology and velocity fluctuations were used to explain the characteristics of the flow. The obtained results summarized as follows.

The instantaneous vorticity fields reveal an unsteady wavy structure of the sphere wake. The concentration of small scale vortices (eddy) is more dominant in the wake of the sphere with the vent than that of the smooth sphere. The PIV results of time-averaged turbulent structures are consistent with the visualized flow field showing the onset of shear layer instability. Time-averaged and instantaneous flow data reveal that the size of the structure of the turbulent flow, the size of wake flow region, the location of singular and double points, the peak values of turbulence quantities as a function of submerged ratio. As observed by dye visualizations, flow structures from the vented sphere exhibit more parallel vortex streets that keeps their form without combination in downstream direction. The shear layer surrounding the recirculation bubble downstream of the sphere has a region of intensified velocity fluctuations with higher values of the variation of Reynolds stress correlations $\left\langle\mathrm{u}^{\prime} \mathrm{v}^{\prime} / \mathrm{U}_{\infty}^{2}\right\rangle$ for the smooth sphere and vented sphere is displayed in Figure 10. Well-defined Reynolds stress patterns due to fluctuations along the shear layers produce a weaker Reynolds stress region very close to the base of the vented sphere case, which occurs as a result of the entrainment of free-stream flow into this wake region. This behaviour of the flow provides the momentum transfer from the free-stream flow into the wake region due to pressure difference between the wake and free-stream flow. The upstream extension of each large-scale cluster occurs further upstream, and the tip of the large-scale clusters is located closer to the base of the 

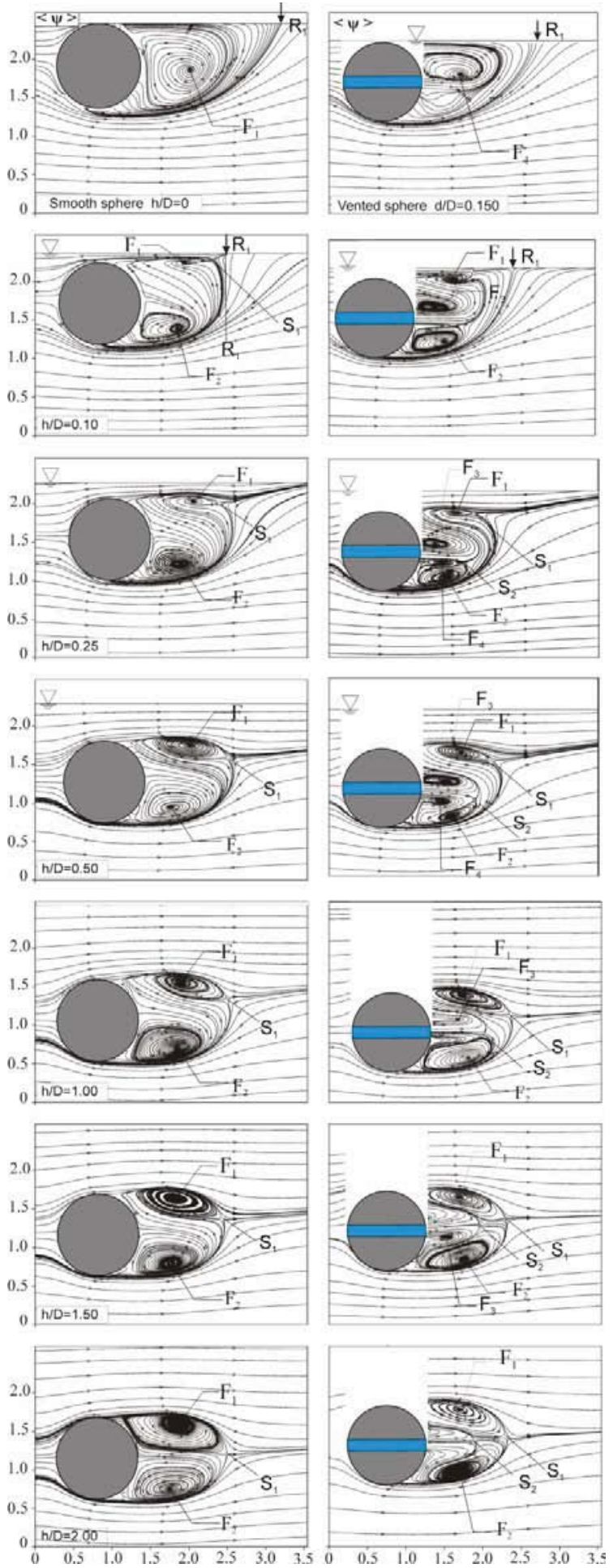

Figure 9: Variation of the streamline patterns $\langle\psi\rangle$ depending on the sphere locations beneath the water free-surface and effect of the ventilation for $\operatorname{Re}=5,000$.
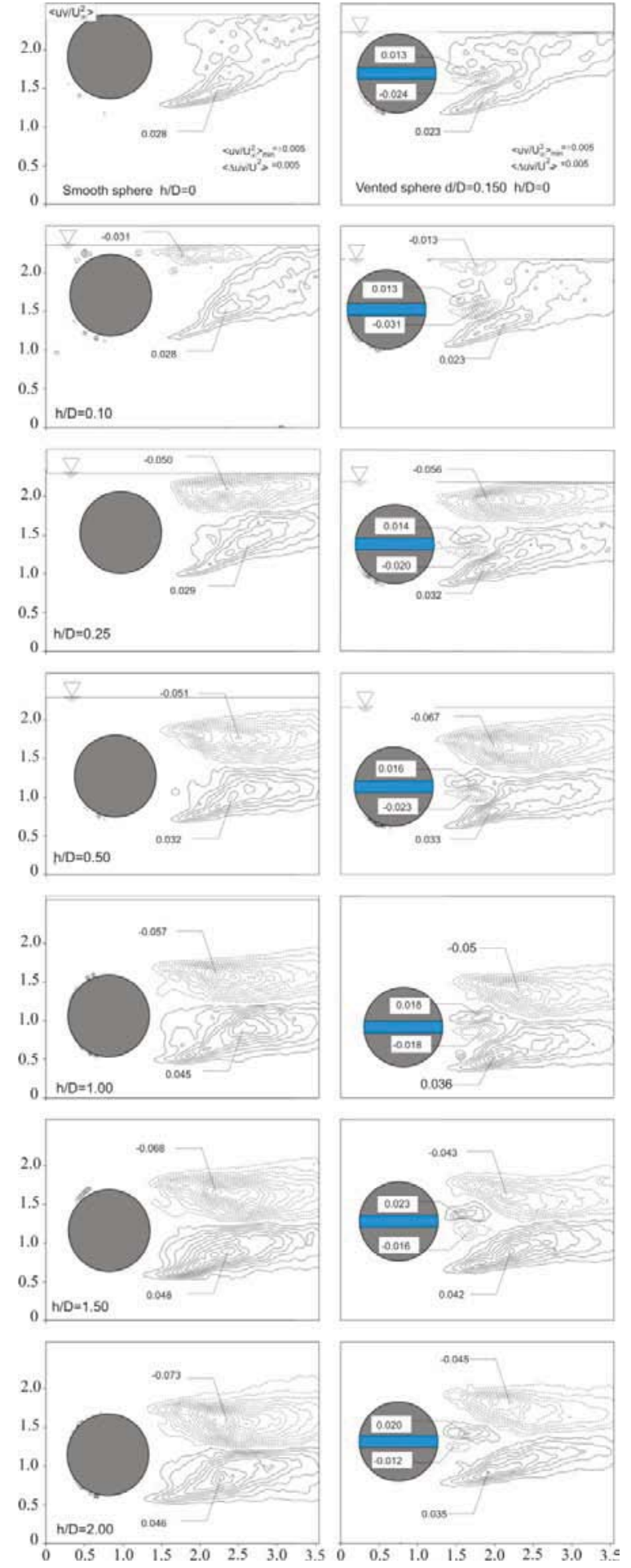

Figure 10: Variation of the Reynolds stress correlation $\left\langle\mathrm{u}^{\prime} \mathrm{v}^{\prime} / \mathrm{U}_{\infty}{ }^{2}\right\rangle$ depending on the sphere locations beneath the water free-surface and effect of the ventilation for $R e=5,000$. 
sphere for all Reynolds numbers as shown in Figure 10. For the vented sphere case, peak values of Reynolds stress correlations are varied considerably due to the effect of the jet flow in the subcritical Reynolds number range. Therefore, surface modifications as in examined in the present study may always results effectively in all cases because there could be a need to retain the structural geometry due to design considerations as well as application of Reynolds number range. The vented sphere model could significantly suppress vortex formation by diffusing the concentrated vorticity in the shear layers behind the body for supercritical Reynolds numbers. Reynolds stress correlations becomes smaller for the smooth sphere case than the vented sphere because of the three-dimensional vortex interactions. However, the concentration of small scale vortices (eddies) is more dominant in the wake of the vented sphere than that of the without vent sphere. Peak values of the larger scale Reynolds scale concentrations generally decrease with increasing submerged ratio as seen all images except $h / D=0.25$ and 0.50 . positive and negative small scale Reynolds Stress correlations occur at the exit region of the vent hole and their magnitudes are always less than half of the larger scale one.

In general, vortex formation lengths for the sphere with the vent are shorter than the smooth sphere case. It is found that the submersed portion of the sphere influences the instability of the vortical flow structure significantly. The shedding location of the largescale vortices rotates slowly and irregularly, and they rotate at random about the stream-wise axis when they travel downstream for both the smooth and vented spheres. The effect of the free surface due to the present of the sphere continue until the $h / D=0.50$. As the distance between the free surface and the bottom shoulder of the sphere increases, the reattachment point to the free surface goes further upstream for $\mathrm{h} / \mathrm{D}=0$ and 0.1 . The flow structure becomes very fluctuating and includes small scale vortices with low velocity distributions in the region between bottom point of the sphere and reattachment point until $h / D>0.5$. Small scale vortices due to the flow through the ventilation magnify the entrainment and circulatory motions between the core wake and free-stream flow regions downstream of the sphere with the vent. When compared to the smooth sphere case, sphere with the vent is found to be better performing as a vortex suppression device even though the Reynolds number is in the subcritical range. The main disadvantage of the vented sphere can be that the hole in the equator of the sphere weakens slightly the body and it might be more expansive from the point of manufacturing process. A small hole like $0.15 \%$ diameter of the bluff body could reduce the drag and fluctuating side forces due to vortex shedding. It is observed that the modified flow structure of the near wake of the vented sphere may be characterized by a pair of counter-rotating ring vortices, which have the effect of aerodynamically streamlining the sphere. The present results can be useful for validating numerical predictions and designers.

\section{ACKNOWLEDGEMENT}

The authors would like to acknowledge the funding of the Scientific and Technological Research Council of Turkey (TÜBİTAK) under contract No:109R028, Coordinatorship of Selcuk University's Scientific Research Project and Scientific Research Projects Office of Cukurova University under contract No: AAP20025. This study is prepared from Sercan Dogan's Master of Science Thesis. 


\section{REFERENCES}

[1] E Achenbach, Vortex shedding from spheres, J. Fluid Mech. 62(2), 1974, pp.209-221.

[2] I Hadzic, V Bakic, M Peric, V Sajn, F Kosel, Experimental and numerical studies of flow around sphere at sub-critical Reynolds number, Eng. Turbul. Model Exp. 5, 2002, pp.667-676.

[3] Y II, J Jang, S J Lee, PIV analysis of near-wake behind a sphere at a subcritical Reynolds number, Exp. Fluids. 44, 2008, Issue 6, 905-914.

[4] A Leder and D Geropp, The unsteady flow structure in the wake of the sphere, SPIE 2052, 1993, pp.119-126.

[5] T Leweke, M Provansal, D Ormie res and R Lebescond, Vortex dynamics in the wake of a sphere, Phys. Fluid, 1999, vol.11, Issue9, p.12.

[6] S Taneda, Experimental investigation of the wake behind a sphere at low Reynolds number, J. Phys. Soc. Japan. 11, 1956, Issue.10, pp.1104-1108.

[7] K Aoki, A Ohike, K Yamaguchi, Y Nakayama, Flying Characteristics and Flow Pattern of A Sphere With Dimples, Journal of Visualization, 2003a, pp.67-76.

[8] J Choi, J Woo-Pyung and $\mathrm{H}$ Choi, Mechanism of Drag Reduction by Dimples on a Sphere, Physics of Fluids 18, 2006, 041702.

[9] J Jeon, J Choi, J Woo-Pyung, C Haecheon and Jınıl P, Active control of flow over a sphere for Drag Reduction at a Subcritical Reynolds number, J. Fluid Mech. vol.517, 2004, pp.113-129.

[10] M Ozgoren, A Okbaz, S Dogan, A Kahraman, R Hassanzadeh, B Sahin and H Akilli, Passive Control of Vortical Flow Structure around a Sphere by an O-ring, Elazıg, $6^{\text {th }}$ International Advanced Technologies Symposium, 2011, ETE-53.

[11] M Ozgoren, B Şahin, E Pınar and Akıllı $H$, Experimental Investigation of Flow structure around a Sphere and Cylinder via Flow Visualization, 5. Ankara International Aerospace Conference, Ankara, 17-19 August 2009, AIAC-2009012.

[12] M Ozgoren, B Şahin, E Pınar and H Akıllı, Comparison of flow structure around sphere and cylinder, $17^{\text {th }}$ National Thermal Science and Technology Conference, Sivas-Turkey, Haziran 2009, pp.73-79, 24-27.

[13] M Ozgoren, A Okbaz, A Kahraman, R Hassanzadeh , B Sahin, H Akilli, S Dogan: Experimental Investigation of the Flow Structure around a Sphere and Its Control with Jet Flow via PIV, Elazıg, $6^{\text {th }}$ International Advanced Technologies Symposium, 2011, ETE-50.

[14] M Ozgoren, E Pinar, B Sahin, H Akilli, Comparison of flow structures in the downstream region of a cylinder and sphere, Int. J. Heat Fluid Flow, 2011, doi: $10.1016 / j$.ijheatfluidflow.

[15] G K Suryanarayana, H Pauer and GEA Meier, Bluff-body drag reduction by passive ventilation, Exp. Fluids 16, 1993, 73-81.

[16] G K Suryanarayana, GEA Meier, Effect of ventilation on the flow field around a sphere, Exp Fluids 19, 1995, pp.78-88.

[17] G K Suryanarayana and A Prabhu, Effect of natural ventilation on the boundary layer separation and near-wake vortex shedding characteristics of a sphere, Exp. Fluids 29, Issue.7, 2000, pp.582-591.

[18] J S Wu and G M Faeth, Sphere wakes in still surroundings at intermediate Reynolds numbers, AIAA J., vol.3, Issue.8, 1993, pp.1448-1455. 
[19] Kim D Yung and $\mathrm{H}$ Choi, Vortical structures behind a sphere at subcritical Reynolds numbers, Phys Fluids 18(1):015102, 2006, doi:10.1063/1.2166454.

[20] S Jeon and H Choi, Suboptimal Feedback Control for Drag Reduction in Flow over a Sphere, Bull. Am. Pbys. Soc., 2005, 50:56.

[21] M M Zdravkovich, Review and classification of various aerodynamic and hydrodynamic means for suppressing vortex shedding, J. Wind Eng Ind. Aerodyn, 7, 1981, pp.145-189.

[22] C Haecheon, J Woo-Pyung and K Jinsung, Control of Flow Over a Bluff Body, Annu. Rev. Fluid Mech., 40, 2008, pp.113-39.

[23] M Gad-el-Hak, Modern Developments in Flow Control, Applied Mechanics Review, vol, V. 49, 1996, pp.365-379.

[24] $P$ Bearman and M Brankovi ' $C$, Experimental studies of passive control of vortexinduced vibration, European Journal of Mechanics B/Fluids 23, 2004, 9-15.

[25] J C Owen and P W Bearman, Passive control of VIV with drag reduction, Journal of Fluids and Structures 15, 2001, pp.597-605.

[26] J Sheridan, J C Lin, D Rockwell, Flow past a cylinder close to a free surface, J. Fluid Mech. 330, 1997, pp.1-30.

[27] T Igarashi, Flow characteristics of a circular cylinder with a vent, Bulletin of the JSME 21, 154, 1978, 656-664.

[28] P W Bearman, The effect of base bleed on the flow behind a two-dimensional model with a blunt trailing edge, The Aero. Ouarterly XVIII, 1967, 207-223. 